\title{
Anomalous autoresonance threshold for chirped-driven Korteweg-de-Vries waves
}

\author{
L. Friedland \\ Racah Institute of Physics, Hebrew University of Jerusalem, Jerusalem 91904, Israel \\ A. G. Shagalov and S. V. Batalov \\ Institute of Metal Physics, Ekaterinburg 620990, Russian Federation \\ and Ural Federal University, Mira 19, Ekaterinburg 620002, Russian Federation
}

(Received 28 August 2015; published 28 October 2015)

\begin{abstract}
Large amplitude traveling waves of the Korteweg-de-Vries (KdV) equation can be excited and controlled by a chirped frequency driving perturbation. The process involves capturing the wave into autoresonance (a continuous nonlinear synchronization) with the drive by passage through the linear resonance in the problem. The transition to autoresonance has a sharp threshold on the driving amplitude. In all previously studied autoresonant problems the threshold was found via a weakly nonlinear theory and scaled as $\alpha^{3 / 4}, \alpha$ being the driving frequency chirp rate. It is shown that this scaling is violated in a long wavelength $\mathrm{KdV}$ limit because of the increased role of the nonlinearity in the problem. A fully nonlinear theory describing the phenomenon and applicable to all wavelengths is developed.
\end{abstract}

DOI: 10.1103/PhysRevE.92.042924

PACS number(s): 05.45.-a, 52.35.Mw, 52.35.Sb

\section{INTRODUCTION}

The Korteweg-de-Vries (KdV) equation

$$
\eta_{t}+\eta_{x}+6 \eta \eta_{x}+\eta_{x x x}=0
$$

is one of the most important equations of nonlinear physics and describes a variety of physical problems, such as shallow water waves, plasma oscillations in a magnetic field, anharmonic lattice oscillations, and more [1,2]. It has a variety of solutions including traveling waves $\eta=\eta(\theta), \theta=k x-\omega t$, the famous solitary waves, and more complex multiphase waves [3,4]. Therefore, the excitation and control of different KdV waves comprises an important goal in a variety of applications. One of the approaches to reaching this goal is based on the idea of autoresonance [5]. The autoresonance is a general property of nonlinear waves and oscillations to stay in resonance with driving oscillations, when the parameters of the system vary in space or time. For example, if instead of Eq. (1) one considers a driven problem

$$
\eta_{t}+\eta_{x}+6 \eta \eta_{x}+\eta_{x x x}=\varepsilon \cos \theta_{d},
$$

where $\theta_{d}=k x-\int \omega(t) d t$, a large amplitude traveling $\mathrm{KdV}$ wave can be excited via autoresonance by starting from zero as a slowly varying driving frequency $\omega(t)$ passes through the linear resonance frequency $\omega_{r}=k-k^{3}$ [6,7]. More generally, a multiphase $\mathrm{KdV}$ wave can be excited and controlled driving the system by a superposition of plane waves with wave vectors corresponding to different spatial harmonics $k_{i}=n_{i} k_{0}$ of the excited KdV solution and driving frequencies $\omega_{i}(t)$ passing simultaneously through the corresponding linear resonance frequencies $\omega_{r_{i}}=k_{i}-k_{i}^{3}[8]$.

One of the most important effects associated with the autoresonance phenomenon is the existence of a sharp threshold on the driving amplitude for transition to autoresonance by passage through a linear resonance. Typically, this threshold scales as $\varepsilon_{t h} \sim \alpha^{3 / 4}$, where $\alpha$ is the driving frequency chirp rate [5]. This threshold was discovered in plasma physics applications [9] and later studied in many fields of physics, ranging from planetary dynamics [10] through nonlinear optics [11] and molecular physics [12] to Josephson junctions [13] and nanomagnets [14]. The autoresonance threshold is also sensitive to the degree of nonlinearity in the driven system. Therefore, the threshold phenomenon can be used in testing approximations in modeling resonant wave excitations. For example, long wavelength ion acoustic waves in plasmas are frequently described by the $\mathrm{KdV}$ equation. This $\mathrm{KdV}$ model is obtained by neglecting all nonlinearities in the underlying fluid equations, but the streaming term $u u_{x}$ ( $u$ being the ion fluid velocity) in the ion momentum equation. Autoresonant excitation of ion acoustic waves was studied recently via the variational theory [15]. It was shown that the autoresonance threshold for excitation of these waves still scaled as $\alpha^{3 / 4}$, but its value deviated significantly from that predicted by the weakly nonlinear $\mathrm{KdV}$ theory. The reason for this deviation was the neglect of additional nonlinear terms in reduction to the KdV model. Here we will show that even within the driven $\mathrm{KdV}$ equation (2), a significant deviation from the usual $\alpha^{3 / 4}$ scaling of the threshold is characteristic of excitation of sufficiently long wavelength traveling waves. We will develop a theory describing this anomalous threshold regime and compare our predictions with numerical simulations. The scope of the paper will be as follows. In Sec. II, we will discuss the passage through resonance in the driven $\mathrm{KdV}$ dynamics and illustrate the anomalous autoresonance threshold in numerical simulations. In Sec. III, the fully nonlinear theory of autoresonant thresholds in the $\mathrm{KdV}$ problem will be developed and compare the results of the theory with numerical simulations. Finally, Sec. IV presents our conclusions.

\section{PASSAGE THROUGH RESONANCE IN DRIVEN KdV DYNAMICS}

We proceed by studying the problem of passage through resonance in Eq. (2). We assume a linear chirp of the driving frequency $\omega(t)=\omega_{r}+\alpha t$, zero initial condition at some large negative $t_{0}$, spatial periodicity, $\eta(x+2 \pi / k, t)=\eta(x, t)$, and solve the driven $\mathrm{KdV}$ equation (2) numerically by using a 


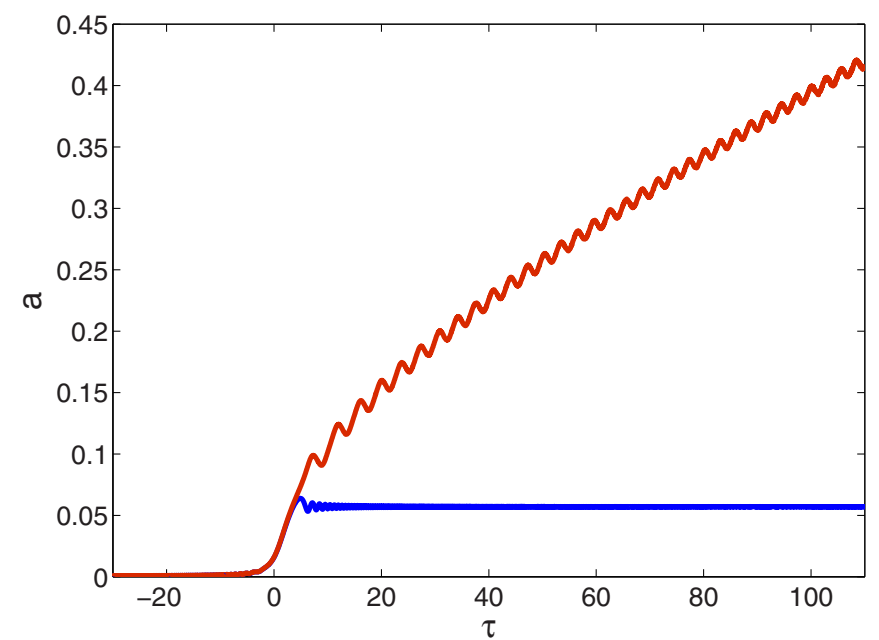

FIG. 1. (Color online) The amplitude of KdV oscillations just below $\left(\epsilon=4.2 \times 10^{-5}\right.$, blue $)$ and above $\left(\epsilon=4.3 \times 10^{-5}\right.$, red $)$ the threshold for transition to autoresonance.

standard spectral method (see, e.g., Ref. [16]). Figure 1 illustrates the threshold phenomenon in transition to autoresonance in the problem and shows the results of numerical simulations for the amplitude $a=(\max \eta-\min \eta) / 2$ of oscillations of $\eta$ versus slow time $\tau=\alpha^{1 / 2} t$, using initial $\tau_{0}=-30$, parameters $k=0.5, \alpha=10^{-5}$, and two values of the driving amplitude $\varepsilon=4.2 \times 10^{-5}$ and $4.3 \times 10^{-5}$ (just below and above the threshold). In Fig. 2 we explore the parameter space and plot the ratio $\varepsilon_{t h} / \varepsilon_{t h}^{0}$ between the numerical threshold driving amplitude for autoresonant transition and that from the weakly nonlinear theory, see Eq. (14) below. One observes large deviations of $\varepsilon_{t h}$ from $\varepsilon_{t h}^{0}$ for smaller $k$ and larger $\alpha$, as well as a significant departure from the usual weakly nonlinear $\varepsilon_{t h} \sim \alpha^{3 / 4}$ scaling [5]. For example, in application to ion acoustic waves in plasmas, our dimensionless $k$ and $\alpha$ correspond to $k \lambda_{D}$ and $\alpha \omega_{p i}^{-2}$ in dimensional notations, where $\lambda_{D}$ and $\omega_{p i}$ are the Debye length and the plasma

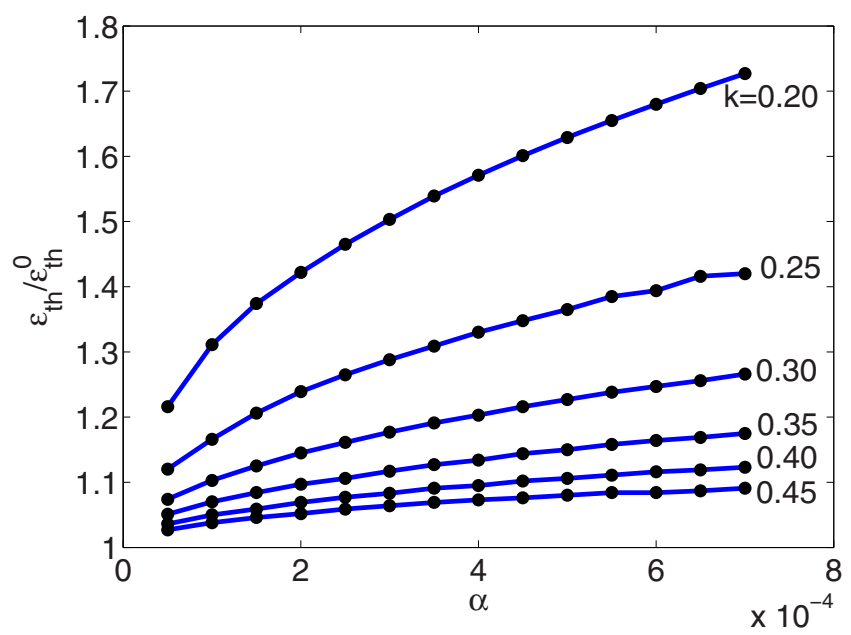

FIG. 2. (Color online) The ratio between the autoresonance threshold to that from the weakly nonlinear theory [Eq. (14)] versus the driving frequency chirp rate $\alpha$. ion frequency. Therefore, as seen in Fig. 2, one can expect a significant deviation of the autoresonance threshold for ion acoustic waves from the weakly nonlinear $\alpha^{3 / 4}$ scaling when (dimensional) $k<0.25 / \lambda_{D}$ and $\alpha>5 \times 10^{-4} \omega_{p i}^{2}$. In this case in plasmas and other systems modeled by the driven $\mathrm{KdV}$ model under similar conditions, higher-order nonlinear effects must be taken into account in explaining the anomalous threshold scaling in the chirped-driven $\mathrm{KdV}$ problem. With this goal in mind, we adopt a fully nonlinear approach to autoresonant KdV dynamics developed in Ref. [7] and based on Whitham's averaged variational principle [17]. We use the conventional representation of the spatial oscillations of $\eta$ as a motion of a quasiparticle in an effective potential of form $V_{\text {eff }}=\eta^{3}-B \eta^{2}+C \eta$ [17]. In the free $(\varepsilon=0)$ system, parameters $B, C$ and the energy $A=\eta_{x}^{2}+V_{\text {eff }}$ of the quasiparticle are constant and can be conveniently expressed in terms of the three turning points $\eta_{1,2,3}$ (numbered in increasing order) satisfying $A-V_{\text {eff }}(\eta)=0$. The amplitude of the free oscillations

$$
a=\left(\eta_{3}-\eta_{2}\right) / 2 \text {. }
$$

is related to the nonlinearity parameter

$$
m=\left(\eta_{3}-\eta_{2}\right) /\left(\eta_{3}-\eta_{1}\right)
$$

via

$$
a=m s / 2,
$$

where $s=2[k K(m) / \pi]^{2}$, while $K(m)$ and later $E(m)$ are the complete elliptic integrals. In a slowly varying driven problem $\eta_{i}$, and, therefore $A, B$, and $C$, and consequently $m$ becomes slow functions of time. The Whitham's averaged variational principle applied to this problem yields the following set of slow evolution equations for $m$ (note $0 \leqslant m \leqslant 1$ ) and phase mismatch $\Phi=\theta-\theta_{d}$ between the driven and driving waves [7]:

$$
\begin{gathered}
m_{t}=-\frac{\varepsilon V}{W} \sin \Phi \\
\Phi_{t}=\alpha t-\Omega-\frac{\varepsilon}{a} \cos \Phi .
\end{gathered}
$$

Here

$$
V=(s / k)[\pi / K(m)]^{2} q /\left(1-q^{2}\right),
$$

where $q=\exp [-\pi K(1-m) / K(m)]$,

$$
W=\frac{2 k^{3} E(m) K(m)[E(m)-K(m)]}{m(m-1) \pi^{4}}[E(m)+(m-1) K(m)],
$$

and the nonlinear frequency shift is

$$
\Omega=k^{3}\left\{1-\left(\frac{2}{\pi}\right)^{2}\left[3 E(m) K(m)+(m-2) K^{2}(m)\right]\right\} .
$$

We have evaluated the autoresonant threshold by solving Eqs. (6) and (7) for the parameters of Fig. 2 and found excellent agreement with full numerical simulations (the difference was within the size of the full circles in Fig. 2), illustrating the validity of the variational theory. 

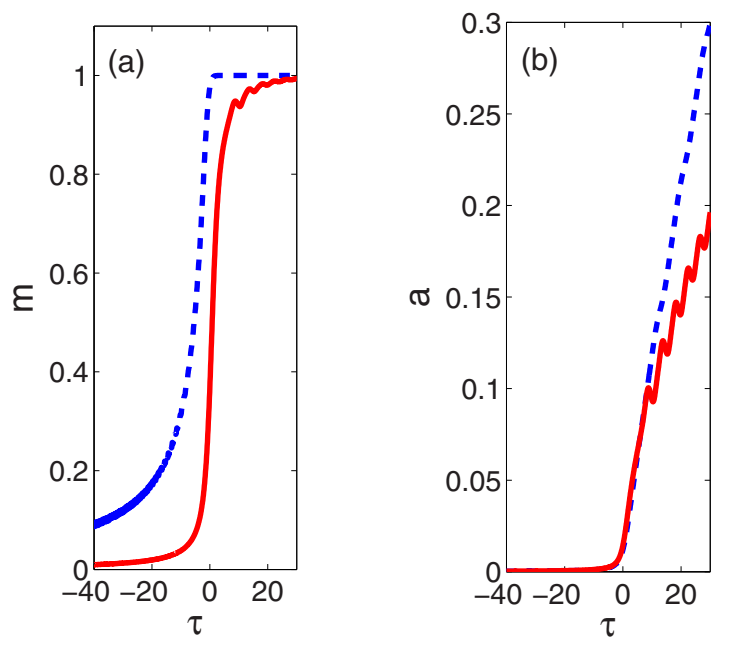

FIG. 3. (Color online) Autoresonant evolution of (a) the nonlinearity parameter and (b) the amplitude of the $\mathrm{KdV}$ wave for $\alpha=1.25 \times 10^{-5}, k=0.106$ (blue dashed lines), and $k=0.354$ (red lines).

We consider the small $m$ limit within the variational approach next. In this case (using lowest significant order series expansions in powers of $m$ ), Eqs. (6) and (7) become

$$
\begin{gathered}
m_{t}=-\epsilon \sin \Phi, \\
\Phi_{t}=\alpha t-\beta m^{2}-\frac{\epsilon}{m} \cos \Phi,
\end{gathered}
$$

where $\epsilon=4 \varepsilon / k^{2}$ and $\beta=3 k^{3} / 32$. This is the standard weakly nonlinear autoresonance problem [5]. The system enters the autoresonant excitation regime provided the driving amplitude $\epsilon$ exceeds the threshold

$$
\epsilon>\epsilon_{t h}^{0}=0.41 \frac{\alpha^{3 / 4}}{\beta^{1 / 2}}
$$

or

$$
\varepsilon_{t h}^{0}=0.335 k^{1 / 2} \alpha^{3 / 4} .
$$

The reason for the deviation of the threshold driving amplitude from $\varepsilon_{t h}^{0}$ as seen in Fig. 2 is the increased role of nonlinearity in the problem for smaller $k$. We illustrate this effect in Fig. 3 in two examples, $k=0.354$ and 0.106 , with $\alpha=1.25 \times 10^{-5}$. The figure shows the phase mismatch $\Phi$, the nonlinearity parameter $m$, and the amplitude $a$ just above the corresponding thresholds $\left(\varepsilon_{t h}=1.25 \times 10^{-4}\right.$ and $\left.1.29 \times 10^{-5}\right)$ as functions of slow time $\tau=\sqrt{\alpha} t$. One can see that the nonlinearity parameter increases rapidly after passing the linear resonance at $\tau=0$ for both values of $k$, but for $k=0.106, m$ becomes close to unity even prior to reaching the linear resonance. This explains the failure of the small $m$ theory for estimating the autoresonance threshold in the problem. We proceed to the fully nonlinear theory of the threshold next.

\section{FULLY NONLINEAR THRESHOLD FOR AUTORESONANT TRANSITIONS}

A surprising observation in Fig. 3 is that in contrast to the nonlinearity parameter $m$, which grows rapidly and approaches
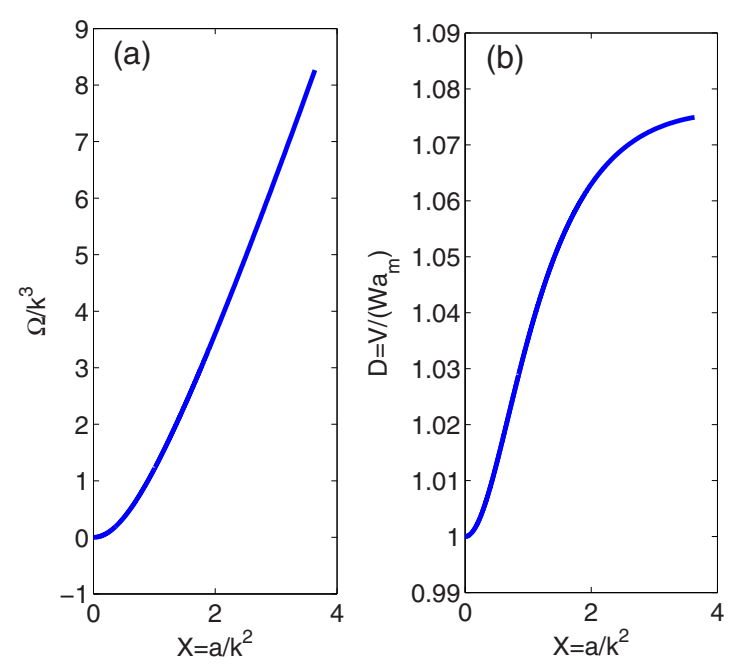

FIG. 4. (Color online) (a) The rescaled frequency $\Omega / k^{3}$ and (b) parameter $D=V /\left(W a_{m}\right)$ versus $X=a / k^{2}$.

unity as one passes the linear resonance, the amplitude $a$ increases slowly and does not exceed 0.2 during the transition to autoresonance even for smaller $k$. This means that the amplitude is the convenient adiabatic variable in the problem and not $m$. To discuss this issue in more detail, we rewrite Eqs. (11) and (12) with $a$ as the dependent variable:

$$
\begin{gathered}
a_{t}=-\frac{\varepsilon V}{W a_{m}} \sin \Phi, \\
\Phi_{t}=\alpha t-\Omega(a)-\frac{\varepsilon}{a} \cos \Phi .
\end{gathered}
$$

Note that (5) and (10) yield a relation

$$
\frac{\Omega}{k^{3}}=F\left(\frac{a}{k^{2}}\right)
$$

and we plot this relation in Fig. 4(a). We also show the coefficient $D=V /\left(W a_{m}\right)$ in Eq. (15) versus rescaled amplitude $X=a / k^{2}$ in Fig. 4(b) (note that $D$ is independent of $k$ ). One can see that both $\Omega$ and $D$ are smooth increasing functions of $X$. However, $D$ is close to unity for all values of $a$ in the transition stage to autoresonance in both examples in Fig. 1. Thus, in studying the autoresonant capture problem, we approximately set $D \approx 1$, introduce the shifted phase mismatch $\Phi^{\prime}=\Phi-\pi$, and rewrite Eqs. (15) and (16) as

$$
\begin{gathered}
X_{t}=\varepsilon^{\prime} \sin \Phi^{\prime}, \\
\Phi_{t}^{\prime}=\alpha t-k^{3} F(X)+\frac{\varepsilon^{\prime}}{X} \cos \Phi^{\prime},
\end{gathered}
$$

where $\varepsilon^{\prime}=\varepsilon / k^{2}$. The autoresonance threshold in this problem can be calculated following a procedure suggested in Ref. [18]. The phase locking in this system proceeds in the linear stage $(t<0)$ of excitation of $X$, where the phase mismatch settles at $\Phi^{\prime} \approx 0$ and $X$ grows as $X \approx \varepsilon^{\prime} /(\alpha|t|)$. If the system remains in autoresonance after passage through the linear resonance, $\Phi^{\prime}$ remains near zero continuously, i.e., the right-hand side of (19) nearly vanishes. Then, we define the quasiequilibrium 
$X_{0}(t)$ satisfying

$$
\alpha t-k^{3} F\left(X_{0}\right)+\frac{\varepsilon^{\prime}}{X_{0}}=0,
$$

approximate $\cos \Phi^{\prime} \approx 1$ in Eq. (19), differentiate this equation in time, and use Eq. (18) to get

$$
\Phi_{t t}^{\prime} \approx \alpha-\varepsilon^{\prime} S\left(X_{0}\right) \sin \Phi^{\prime}
$$

where

$$
S\left(X_{0}\right)=k^{3} \frac{d F}{d X_{0}}+\frac{\varepsilon^{\prime}}{X_{0}^{2}} .
$$

Equation (21) describes a pendulum having a slowly varying frequency $v(t)=\left[\varepsilon^{\prime} S\left(X_{0}\right)\right]^{1 / 2}$ under the action of a constant torque $\alpha$. Therefore, after the capture into resonance, bounded adiabatic oscillations of $\Phi^{\prime}$ (corresponding to phase locking and autoresonance in our original problem) will be sustained, provided the time variation of $v(t)$ is sufficiently slow and

$$
\alpha<\varepsilon^{\prime} S\left[X_{0}(t)\right] .
$$

We will see below that $S\left(X_{0}\right)$ has a minimum at some value of $X_{0}=\bar{X}$. Consequently, the threshold chirp rate for a continuing autoresonance in our system is

$$
\alpha_{t h}=\varepsilon^{\prime} S(\bar{X})=\frac{\varepsilon}{k^{2}} S(\bar{X}) .
$$

By definition, $\bar{X}$ satisfies

$$
\bar{X}^{3} \frac{d^{2} F}{d \bar{X}^{2}}=P,
$$

where we defined a new parameter

$$
P=2 \varepsilon^{\prime} / k^{3}=2 \varepsilon / k^{5} .
$$

Thus, $\bar{X}=\bar{X}(P)$ and, by substitution of (25) into (24), we get

$$
\alpha_{t h}=k \varepsilon\left(\frac{d F}{d \bar{X}}+\frac{1}{2} \bar{X} \frac{d^{2} F}{d \bar{X}^{2}}\right) \equiv k \varepsilon R(P) .
$$

We plot $R(P)$ as well as the associated nonlinearity parameter $m[\bar{X}(P)]$ in Fig. 5. One can see in the figure that parameter $P$ characterises the strength of the nonlinearity in the problem. Next, we consider the small and large $P$ limits, both allowing analytic evaluation of the threshold.

In the weakly nonlinear case [7],

$$
F=1.5 X^{2} \text {. }
$$

Then $\bar{X}=(P / 3)^{1 / 3}$ and

$$
R=4.5\left(\frac{P}{3}\right)^{1 / 3}
$$

which after substitution into (26), yields

$$
\alpha_{t h}=4.5 k \varepsilon(P / 3)^{1 / 3}=3\left(\frac{3}{2 k}\right)^{2 / 3} \varepsilon^{4 / 3},
$$

or

$$
\varepsilon_{t h}=3^{-5 / 4} \sqrt{2 k} \alpha^{3 / 4}=0.36 \sqrt{k} \alpha^{3 / 4} \text {. }
$$

The last result differs by $9 \%$ only from our original weakly nonlinear threshold $\varepsilon_{t h}=0.41 \sqrt{2 k / 3} \alpha^{3 / 4}$ [see Eq. (13)].

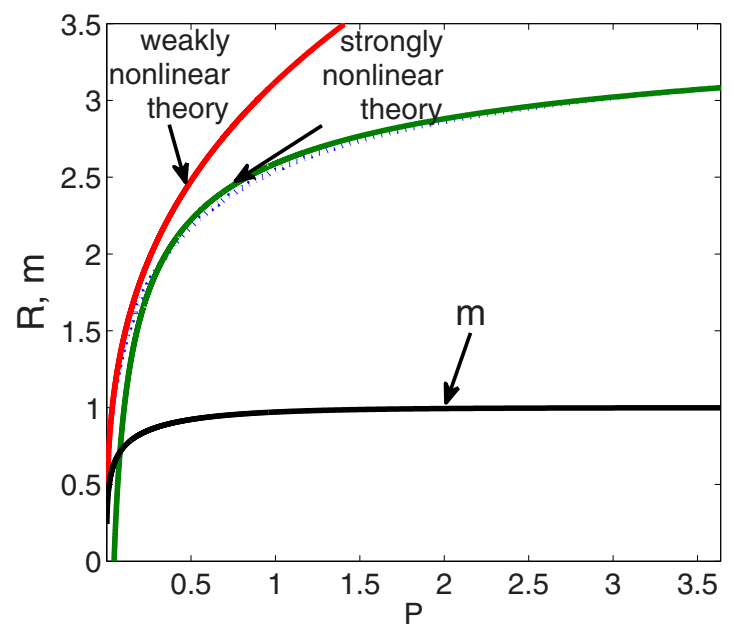

FIG. 5. (Color online) The function $R$ (dotted blue line) and nonlinearity parameter $m$ (black line) versus parameter $P$. The weakly and strongly nonlinear approximations for $R$ are represented by the red and the green lines, respectively.

In the strongly nonlinear regime, $m \approx 1$, and [7],

$$
F=1-4\left(\frac{3}{\pi} X^{1 / 2}-X\right) .
$$

This yields $\bar{X}=(\pi P / 3)^{2 / 3}$ and

$$
R=4-\frac{9}{2 \pi}\left(\frac{3}{\pi P}\right)^{1 / 3} .
$$

Therefore,

$\alpha_{t h}=k \varepsilon\left[4-\frac{9}{2 \pi}\left(\frac{3}{\pi P}\right)^{1 / 3}\right]=k \varepsilon\left[4-\frac{9 k}{2 \pi}\left(\frac{3 k^{2}}{2 \pi \varepsilon}\right)^{1 / 3}\right]$.

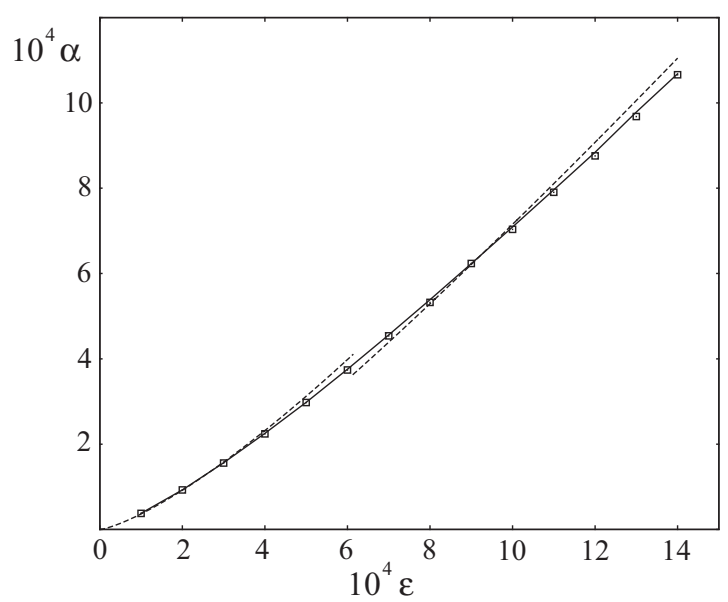

FIG. 6. The numerical threshold chirp rate $\alpha_{t h}$ versus the driving parameter $\varepsilon$ (full line) in $k=0.353$ case. The dashed line represents the results from the weakly nonlinear (for $\varepsilon<6.2 \times 10^{-4}$ ) and strongly nonlinear theories (for $\varepsilon>6.2 \times 10^{-4}$ ), the solid line is obtained by using Eqs. (6), (7), and the squares are the results of the KdV simulations. 
We compare the limiting analytic results (28) and (32) for $R(P)$ with numerics in Fig. 5. Remarkably, to a good approximation, one can use the following unifying formula for calculating $R$ for all values of $P$ :

$$
R=\left\{\begin{array}{cc}
4.5\left(\frac{P}{3}\right)^{1 / 3}, & P<0.225 \\
4-\frac{9 k}{2 \pi}\left(\frac{3}{\pi P}\right)^{1 / 3}, & P>0.225 .
\end{array}\right.
$$

Consequently,

$$
\alpha_{t h}=\left\{\begin{array}{c}
3\left(\frac{3}{2 k^{\prime}}\right)^{2 / 3} \varepsilon^{4 / 3}, \quad \varepsilon<0.112 k^{5}, \\
k \varepsilon\left[4-\frac{9 k}{2 \pi}\left(\frac{3 k^{2}}{2 \pi \varepsilon}\right)^{1 / 3}\right], \quad \varepsilon>0.112 k^{5} .
\end{array}\right.
$$

As an illustration, Fig. 6 compares this result with those obtained by solving adiabatic Eqs. (6) and (7) (solid line) and with the full numerical simulations (squares) in the case $k=0.353$. The values of $\varepsilon$ in the figure cover both the small and large $P$ approximations in this example.

\section{CONCLUSIONS}

In conclusion, we have studied the problem of anomalous scaling of the threshold for transition to autoresonance in excitation of large amplitude traveling $\mathrm{KdV}$ waves. The anomaly characterized the long wavelength limit and was explained by the increased role of nonlinearity in passage through the linear resonance. As the result, the threshold driving amplitude departed from the $\alpha^{3 / 4}$ scaling with the driving amplitude chirp rate characteristic of all previously studied autoresonant problems. We have developed a fully nonlinear theory describing the autoresonant threshold KdV anomaly. The theory was based on the Whitham's averaged variational principle for driven waves [7] and yielded the analytic result for the threshold in the KdV problem valid uniformly for arbitrary wavelengths. The theory was in an excellent agreement with simulations. We expect a similar increased role of the nonlinearity in the problem of autoresonant excitation of more complex multiphase $\mathrm{KdV}$ waves, as well as in other related resonantly driven nonlinear systems. For example, in the small wavelength limit, the nonlinear Schrödinger (NLS) equation reduces to the $\mathrm{KdV}$ equation [19] and, therefore, in some parameter regime, the autoresonant threshold anomaly should characterize the driven NLS problem as well. For similar reasons, a fully nonlinear theory of the autoresonance thresholds may be needed in studying autoresonant Toda chain excitations [20]. More generally, searching for anomalous autoresonant transition regimes in driven few or many degrees of freedom (and continuous) systems seems to be an important goal for future research.

\section{ACKNOWLEDGMENTS}

This work was supported by the Israel Science Foundation (Grant No. 30/14) and, partially, by FASO 01201463332 and UDRAS Project No. 15-8-2-7.
[1] V. I. Karpman, Nonlinear Waves in Dispersive Media (Pergamon, Oxford, 1975).

[2] A. Scott, Nonlinear Science: Emergence and Dynamics of Coherent Structures (Oxford University Press, New York, 1999).

[3] S. Novikov, S. V. Manakov, L. P. Pitaevskii, and V. E. Zacharov, Theory of Solitons (Consultants Beureau, New York, 1984).

[4] M. J. Ablowitz and H. Segur, Solitons and Inverse Scattering Transform (SIAM, Philadelphia, 1981).

[5] L. Friedland, Scolarpedia 4, 5473 (2009).

[6] I. Aranson, B. Meerson, and T. Tajima, Phys. Rev. A 45, 7500 (1992).

[7] L. Friedland, Phys. Plasmas 5, 645 (1998).

[8] L. Friedland and A. G. Shagalov, Phys. Rev. Lett. 90, 074101 (2003).

[9] J. Fajans, E. Gilson, and L. Friedland, Phys. Rev. Lett. 82, 4444 (1999).

[10] L. Friedland, Astrophys. J. 547, L75 (2001).
[11] A. Barak, Y. Lamhot, L. Friedland, and M. Segev, Phys. Rev. Lett. 103, 123901 (2009).

[12] G. Marcus, L. Friedland, and A. Zigler, Phys. Rev. A. 69, 013407 (2004).

[13] K. W. March, R. Vijay, I. Barth, O. Naaman, J. Aumentado, L. Friedland, and I. Siddiqi, Nature Phys. 7, 105 (2011).

[14] G. Klughertz, P.-A. Hervieux, and G. Manfredi, J. Phys. D: Appl. Phys. 47, 345004 (2014).

[15] L. Friedland and A. G. Shagalov, Phys. Rev. E 89, 053103 (2014).

[16] C. Canuto, M. Y. Hussaini, A. Quarteroni, and T. A. Zang, Spectral Methods in Fluid Dynamics, Springer Series in Computational Physics (Springer, Berlin, 1988).

[17] G. B. Whitham, Linear and Nonlinear Waves (Wiley, New York, 1974), pp. 569-570.

[18] J. Fajans and L. Friedland, Am. J. Phys. 69, 1096 (2001).

[19] Yu. Kivshar and B. Luther-Davies, Physics Reports 298, 81 (1998).

[20] M. Khasin and L. Friedland, Phys. Rev. E 68, 066214 (2003). 